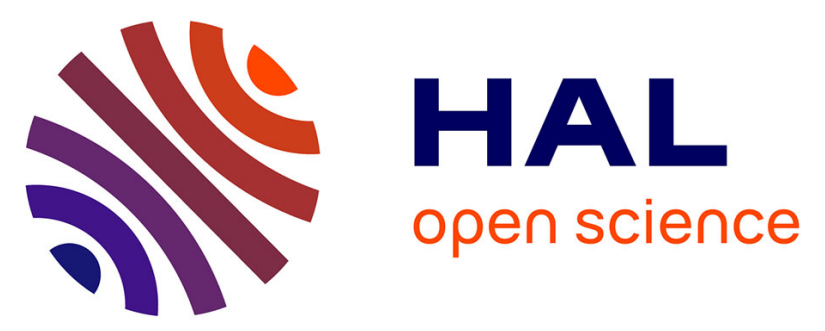

\title{
MorphoScript: a dedicated analysis to assess the morphology and contractile structures of cardiomyocytes derived from stem cells
}

Tess Homan, Hélène Delanoë-Ayari, Albano C Meli, Olivier Cazorla, Csilla Gergely, Alexandre Mejat, Philippe Chevalier, Adrien Moreau

\section{To cite this version:}

Tess Homan, Hélène Delanoë-Ayari, Albano C Meli, Olivier Cazorla, Csilla Gergely, et al.. MorphoScript: a dedicated analysis to assess the morphology and contractile structures of cardiomyocytes derived from stem cells. Bioinformatics, 2021, 10.1093/bioinformatics/btab400 . hal-03258544

\author{
HAL Id: hal-03258544 \\ https://hal.science/hal-03258544
}

Submitted on 14 Jun 2021

HAL is a multi-disciplinary open access archive for the deposit and dissemination of scientific research documents, whether they are published or not. The documents may come from teaching and research institutions in France or abroad, or from public or private research centers.
L'archive ouverte pluridisciplinaire HAL, est destinée au dépôt et à la diffusion de documents scientifiques de niveau recherche, publiés ou non, émanant des établissements d'enseignement et de recherche français ou étrangers, des laboratoires publics ou privés. 


\title{
MorphoScript: a dedicated analysis to assess the morphology and contractile structures of cardiomyocytes derived from stem cells
}

\author{
Tess Homan ${ }^{1}$, Hélène Delanoë-Ayari ${ }^{1 *}$, Albano C. Meli ${ }^{2}$, Olivier Cazorla ${ }^{2}$, \\ Csilla Gergely ${ }^{3}$, Alexandre Mejat ${ }^{4}$, Philippe Chevalier ${ }^{4,5}$ and Adrien Moreau ${ }^{2}$ \\ 1 Claude Bernard University, Lyon 1, Université de Lyon, Lyon; Institut lumière matière (Lyon, France), 2 PhyMedExp, INSERM, CNRS, University of \\ Montpellier, Montpellier, 3 L2C, University of Montpellier, CNRS, Montpellier, France, 4 Claude Bernard University, Lyon 1, Université de Lyon, Lyon; \\ Neuromyogene Institut (Lyon, France), 5 Hospices civils de Lyon, Service de Rythmologie (Bron, France).
}

*To whom correspondence should be addressed.

\begin{abstract}
Motivation: Cardiomyocytes derived from stem cells are closely followed, notably since the discovery in 2007 of human induced pluripotent stem cells (hiPSC). Cardiomyocytes (hiPSC-CM) derived from hiPSC are indeed more and more used to study specific cardiac diseases as well as for developing novel applications such as drug safety experiments. Robust dedicated tools to characterize hiPSC-CM are now required. The hiPSC-CM morphology constitutes an important parameter since these cells do not demonstrate the expected rod shape, characteristic of native human cardiomyocytes. Similarly, the presence, the density and the organization of contractile structures would be a valuable parameter to study. Precise measurements of such characteristics would be useful in many situations: for describing pathological conditions, for pharmacological screens or even for studies focused on the hiPSC-CM maturation process.

Results: For this purpose, we developed a MATLAB based image analysis toolbox, which gives accurate values for cellular morphology parameters as well as for the contractile cell organization.

Implementation: To demonstrate the power of this automated image analysis, we used a commercial maturation medium intended to promote the maturation status of hiPSC-CM, and compare the parameters with the ones obtained with standard culture medium, and with freshly dissociated mouse cardiomyocytes.

Contact: Helene Delanoe-Ayari, Ph.D., Institut Lumiere Matiere, UMR5306 - UCBL - CNRS, 10 rue Ada Byron, 69622 Villeurbanne CEDEX, France, Email: helene.delanoe-ayari@univ-lyon1.fr
\end{abstract}

\section{Introduction}

Cardiovascular troubles are one of the main causes of death worldwide. The identification of new pathological mechanisms as well as the elaboration of efficient and specific therapeutics remains challenging, notably since molecular defects are still poorly understood. Appropriate tools to investigate pathological mechanisms, to develop new therapeutics and to ensure the pharmacovigilance process are critically lacking in the cardiovascular area. Most of the studies are so far performed using either heterologous expression systems or transgenic animal models that besides great value also present large limitations. Human induced pluripotent stem cells (hiPSC) were first described in 2007 by Takahashi and coworkers (Takahashi, et al., 2007). Since then, human fibroblasts, peripheral blood mononuclear cells, or even cells in urine have been reprogrammed into hiPSC (Agu, et al., 2015; Sochacki, et al., 2016; Takahashi, et al., 2007). This major breakthrough along with cardiomyocyte (hiPSC-CM) differentiation protocols considerably increased fundamental research as 
well as drug testing opportunities. Since 2013, confluent monolayers of spontaneously beating hiPSC-CM can be obtained in slightly more than a week using the Wnt signaling pathway modulation (Burridge, et al., 2014; Lian, et al., 2013). This novel model demonstrates several great advantages considering that hiPSC cells can be derived from healthy individuals or from patients and constitute one more step towards personalized medicine. hiPSC-CM can be highly representative of the patient's genetic background, but most of all, hiPSC-CMs constitute an unlimited source of human cardiac cells, extremely valuable considering that native human cardiac tissues are rarely available. The study of specific proteins is realized in a "human cardiac intracellular environment". Indeed, hiPSC-CM demonstrate a functional electrical activity, the expression and function of contractile proteins, as well as specific cardiac metabolic functions (Kadari, et al., 2014; Moreau, et al., 2017; Moreau, et al., 2016; Sleiman, et al., 2020; Tohyama, et al., 2013). However, as all experimental models, hiPSC-CM have several limitations. For example, when compared with native human cardiomyocytes, some differences in their electrical activity (i.e. action potentials) have been reported (Bett, et al., 2013; Denning, et al., 2016; Guo and Pu, 2020). Another marker of hiPSC-CM immaturity is the cellular morphology and their contractile protein assembly since the typical rod shape of human cardiomyocytes is not usually reported (Acimovic, et al., 2018; Denning, et al., 2016; Lundy, et al., 2013; Moreau, et al., 2017; Moreau, et al., 2018; Moreau, et al., 2016; Pasqualin, et al., 2015; Ribeiro, et al., 2015; Sleiman, et al., 2020). The field of hiPSC-CM is currently under intense investigation and many efforts are made to use these cells in pharmacological development or safety drug testing (Fermini, et al., 2015). Furthermore, since hiPSC-CM are spontaneously beating, their morphology and contractile properties are important markers of their health and function. However, while powerful method to measure sarcomere parameters exist (Gannier, et al., 1993; Guo and Song, 2014; Pasqualin, et al., 2015), due to the novelty of hiPSC-CM model, efficient dedicated analysis tools often lack and need to be developed for quantifying the level of organization of sarcomeres which are the essential blocks for the beating of the monolayers. Measurements of the degree of alignments, their spacing, their concentration in proteins are needed for a good characterization of the cell properties in different culture conditions.

Here we describe the MorphoScript, a user friendly versatile automatic analysis tool specifically designed to characterize the hiPSC-CM morphology as well as the organization of their contractile structures. After the automatic detection of hiPSC-CM edges and of contractile protein organization, the Fast Fourier Transform is used to provide information on the organization of the contractile structures. To illustrate the functions of MorphoScript, we used the Pluriomics $(\mathbb{B})$ medium to improve the maturation state of our hiPSC-CM. We are convinced that MorphoScript, a hiPSC-CM dedicated analysis tool will constitute a highly valuable tool in many studies from fundamental research to pharmacological development or drug safety testing.

\section{Methods}

A detailed experimental methods section is available in the online supplementary material.

\section{Using MorphoScript:}

MorphoScript was created using MATLAB R2017a. MorphoScript is freely available upon request to the authors. MorphoScript starts by looking at the overall shape of the cell, followed by an in-depth analysis of the protein organization.
The analysis rely on the fact that sarcomeres can be clearly identified on the images, as the typical spacing between sarcomeres is around $\sim 2 \mu \mathrm{m}$. This means that we will need a resolution of at least $0.5 \mu \mathrm{m}$ to respect the Shannon criterion. This will be ensured with a good objective with a numerical aperture of at least 0.7 . The magnification can be then chosen according to the available camera pixel size and tube length magnification the aim being to get at least $0.5 \mu \mathrm{m}$ per pixel. Of course, higher pixel resolution would be even better, we mainly used here 0.06 $\mu \mathrm{m} /$ pixels.

There is no enforcement on the type of microscopy to be used, it can be either a regular widefield fluorescent microscope, a confocal microscope, spinning disk. Of course, the background should be small enough for ensuring a proper visualization of the sarcomeres, this means that a signal/noise ratio should be at least higher than 2 .

Respecting this criterion should ensure a proper Fourier Analysis result. However, the very way to check that the image quality is good enough is to look at the Fourier transform image where, by eye, we clearly identify a good sarcomeres' organization, and the image should resembles the one showed on Fig1. (i.e. small stripes). If not, this means will have to increase resolution or signal/noise ratio.

As a first step in characterizing the shape of the cell the cell is cut out from its environment (Figure 1). In the ideal case where a single cell is imaged in front of a homogeneous background this can be done automatically, otherwise a semi-automatic or manual method is used. For the manual case the user traces the outline of the cell with a mouse, adding as many points as desired to form a close fitting mask. To automatically create a mask the image is filtered using an entropy filter, and subsequently thresholded into a black and white image using an Otsu threshold (Figure 1).

The black and white mask -cell mask- is improved by removing components with a connectivity of 8 that are smaller than 1000 pixels, followed by closing the image with a 10x10 disk kernel.

A semi-automatic mode is available where the crude outline of the cell is drawn by hand, but the precise circumference is calculated using the automatic method. With this program one or more cells can be cut out per image and for every cell a mask is formed (Figure 1). Most cell outlines can be found automatically, only cells that touch each other need an experts' eye to separate them.

From this mask several parameters are calculated (See supplementary Figure 1).

Rectangularity and elongation are calculated using the bounding box of the mask (smallest rectangular box that can be fitted around the mask of the cell).

The rectangularity is defined as the area of the cell over the area of the bounding box. Perfectly rectangular cells have a rectangularity of 1 which decreases if the cell becomes more irregular or rounder.

The elongation is one minus two times the length of the short side of the box over the long side of the bounding box. The most elongated cells will present an elongation of one and the round ones an elongation of 0 . These two values are closely related to the compactness (the perimeter squared over 4 times pi times the surface area). The last parameter is calculated using a fit of the mask by an ellipse that has the same secondmoments as the region defined by the mask: ellipticity is the ratio of $\frac{l_{m}}{l_{M}}$, where $l_{m}$ and $l_{M}$ represent respectively the small and large axes of the ellipse. For this, we use the "regionprops" function of MATLAB.

In the next step, the image intensity of the cell is evaluated. Another automatic Otsu threshold is applied to find the parts of the cell that are 
covered by proteins, resulting in a second mask -protein mask- as displayed in the lower image in (Figure 1 and Supplementary Figure 1).

Then, using the two masks now obtained (cell + protein one), the coverage is defined as the number of white pixels in the protein mask, divided by the number of white pixels in the cellmask). The mean intensity of the protein fluorescence under the protein mask $I_{p \text {,raw }}$ as well as the mean background of fluorescence intensity under the cell but outside of the sarcomeres patterns, $I_{b}$ are stored. We define the protein intensity as reported in Table 1 , as $\frac{I_{p, r a w}}{I_{b}}$, for normalization and for correcting for different illumination levels.

One can have a look at supplementary Figure 1, for getting a glance on the user interface used to create the mask and for getting the shape parameters

The orientation of the stripes can also be analyzed. The image containing the entire cell is cut up into squares of $L^{*} \mathrm{~L} \mu \mathrm{m}$ (Note that the scale is automatically detected or asked to the user if this parameter was not saved during image acquisition). The window size $\mathrm{L}$ is a crucial parameter in the analysis: a smaller window leads to a more resolved analysis as only the average orientation in a window is determined. But L should be big enough to contain at least 5 contractile structures to ensure a robust and reliable orientation result (see (Durande, et al., 2019) for details on this limit). The window size $\mathrm{L}$ has to be set by the user. However, the final results should be relatively independent of $\mathrm{L}$, as long as the lower limit (five wavelengths) is obeyed, and the window size is not too big. In the software, if the pixel size is available, the default value for $\mathrm{L}$ is $15 \mu \mathrm{m}$, this ensures at least typically 7 sarcomeres in the interrogation window. It is advised to vary $\mathrm{L}$ for a representative test image and find the window size for which small changes have no significant effect on the final calculated parameters."

On each of these sub-areas a 2-D Fourier transform is calculated as depicted in supplementary Figure 2.

Before applying a fast discrete Fourier transform on the data, we first decompose the images in periodic (p), plus smooth components (s), and apply the transform on $p$ to avoid edge effects artefacts (Moisan, 2010). The periodic component $p$ looks like the original image, except that its Discrete Fourier Transform avoids the classical "cross structure" artifact. For discrete signals, a Discrete Fourier Transform (DFT) is computed using a Fast Fourier Transform (FFT) algorithm.

The Fourier transform $(\mathrm{X})$ of a $2 \mathrm{D}$ image $(\mathrm{x})$ of size $\mathrm{N}$ is given by:

$$
X(s+1, t+1)=\sum_{n=0}^{N-1} \sum_{m=0}^{N-1} e^{-\frac{2 i \pi s n}{N}} e^{-\frac{2 i \pi s m}{N}} x(n+1, m+1)
$$

We then calculate the spectral density energy given by $|X(s, t)|^{2}$. The result is a $2 \mathrm{D}$ matrix and

after a fftshift the distance to the center (brightest pixel in the middle of the image stack) corresponds to the radial frequency. We set this centered value (which corresponds to the mean intensity in the image) to zero and then normalize our signal and compute

$$
e_{n}(s, t)=\frac{|X(s, t)|^{2}}{\sum_{s=1}^{N-1} \sum_{t=1}^{N-1}|X(s, t)|^{2}}
$$

When a sarcomere pattern is present in the window of interest, it resembles the one observed in Figure 1: i.e. an array of parallel lines. The aim of the analysis is to find the orientation of these lines which is orthogonal to the orientation of the sarcomeres. From the distance in between the lines the sarcomeres spacing can be calculated.
The angle is calculated by summing the intensities of all pixels on a radial line in the Fourier spectrum. The angle with the highest sum represents the angle parallel to a single unit sarcomere in the original image (see Supplementary Figure 2 to see an example of this type of curve showed by the software interface).

Sometimes it is hard to distinguish between the main angle and the orthogonal angle because a repetitive and intense pattern is also present in the orthogonal directions. In those cases, we apply an additional test investigating the variation along this radial line.

The center pixels are cut out because they contain noise coming from the windowing effect when performing Discrete Fourier Transform. In Figure 2, the angle for every sub-image is drawn using a pink line. The value of the total power of the Fourier spectrum on the maximum angle is a good measure for the amount of order in the image.

If a clear repetitive pattern is present in this particular sub-image the power is high, whereas more homogeneous sub-images do not possess a clear directionality and have a corresponding low power for the main direction.

The power threshold above which the sub-area is defined as ordered is called the order threshold. This is an important parameter that has to be set by the user.

In Figure 2, this is indicated by solid (order) and dashed (no-order) lines. The angle represents the main direction for the sub-area.

We define an order parameter, which is the percentage of sub-areas in the image above the threshold.

In cells where almost the entire surface is covered by fluorescent signal and all sub-areas contain structured patterns. The order parameter is in that case defined as 1 . Cells where only a portion of the surface is covered with a striated pattern have a lower order parameter.

One characteristic of cardiac cells is that all the sarcomeres are aligned. It is an important feature to quantify in hiPSC-CM.

For this purpose, we measure the distribution of angles in each cell (see polar histograms on Figure 2). The orientation distribution is defined as the standard deviation of all the angles found in the cell (only including sub-areas with an order above the order threshold).

To capture a higher amount of detail the sub-areas can overlap, increasing the number of processed regions and the statistics of the analysis.

This analysis procedure runs fully automatic, with only three input parameters: the window size, window's overlap and an order threshold value.

The order threshold value is globally independent of image size and intensity and is therefore universal for experiments performed at different conditions or even in different labs.

The influence of the choice of window size and window overlap is minimal as long as the bottom limit of at least five wavelengths are seen in the field of view.

We have found that a small portion of the obtained angles do not correspond to the angles we would have chosen by hand, for highly accurate results on low number of samples, we recommend to correct manually these few windows, which is very straightforward with Morphoscript. Note that each time an angle correction is made and saved, this implicitly means that the user identifies a structure in the sub-area and whatever the value of the Fourier peak (above/or below the threshold), this area is now counted as organized.

Once the angle is determined, it is possible to determine the spacing in between the sarcomeres. For that, we plot the intensity in the Fourier 
Transform Image along a line perpendicular to the main direction found. On this line, the position of the first intense maximum is a measurement of the spacing. A graph of the intensity along this line is shown in the user interface (Supplementary material Figure 2B), and manual correction could be done if the maximum found is not the appropriate one.

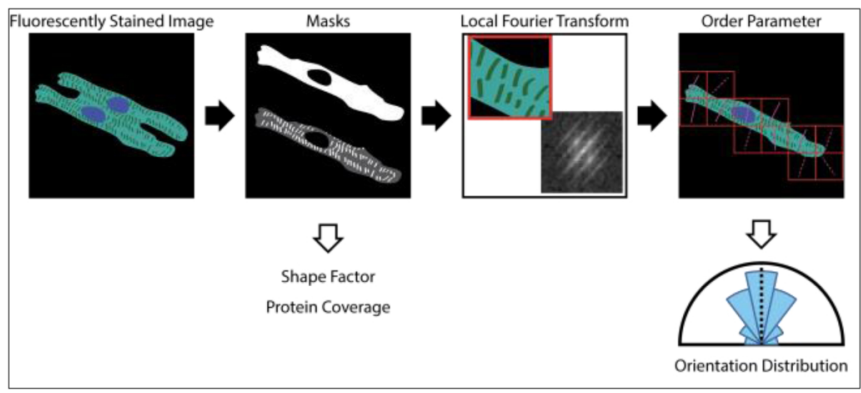

Fig. 1. Analysis pathway of the MorphoScript tool. Cells used present a sarcomeric organization stained either by a specific antibody (actinin, myosin-light chain...) or marker free techniques such as Second Harmonic generation. First, cells contours are defined, and a mask is calculated. Protein coverage under the mask and shape factors are calculated. Then the area under the cell is divided in sub-areas on each of which a local discrete Fourier is performed. The analysis of the Fourier signal enables to get the local orientation of the sarcomeres and their structure

\section{Results}

\subsection{The MorphoScript analysis:}

Morphoscript is a dedicated software for analyzing the contractile structures of cardiomyocytes to extract relevant figures enabling the comparison in between conditions. It is based on the analysis of images where these structures are clearly identifiable by eyes. It can be a greyscale or color image where one of the channels corresponds to the fluorescent signal of a protein of interest present in sarcomeres (antibodies or Second Harmonic Signal) (Figure 2). The goal will be to quantify the organization of the sarcomeres in the images.

Morphoscript starts with a user-friendly interface used to select files to analyze (Supplementary Figure 3). Next, the saving folder has to be set. In case of absence of dedicated saving folder, the script will automatically create a new folder.

The analysis then begins with the creation of a mask for each cell to be analyzed. Each image can contain multiple cells to analyze. This is a crucial step meant to determine the cell borders. For this purpose, 3 methods are provided: a manual way (using mouse), an automated way (using an entropy filter and automatic threshold (see methods) and a combination method that will automatically search for cell borders inside an area determined by the user (Figure 1). The analysis can be aborted after the masks creation. In such case, the user can later automatically search for available masks and resume the analysis without creating new ones.

The analysis is then separated into 2 distinct processes: 1) the cellular shape analysis; 2) the striation analysis.
The cell shape analysis (parameters shown Table 1) is based on the cell edges identified through the first step (cell masks creation). The capacity of a cell to contract is directly linked to the presence of contractile proteins. The part of the cell covered by proteins is thus evaluated using the creation of a protein mask (see method for details, and the two masks created in Figure 1). Next to the number of fluorescent pixels, we can also look at the intensity of the pixels. To get rid of an eventual difference in lighting and exposure settings, we normalize the intensity of the protein by the mean intensity of the cell's background (see methods). The value obtained is the normalized protein intensity as shown in Table 2. There are several ways to describe the overall shape of the cell. For this purpose, the cell mask alone is used. The compactness, rectangularity, elongation and ellipticity are calculated (Supplementary Figure 1 and Table 1).

The organization and orientation of sarcomeric labelling can also be evaluated. To quantify these parameters, the cell is cut into small subareas. On each of these sections a Fourier analysis is performed to determine if there is order in that specific region. If an image depicts a repetitive pattern, the characteristics of this pattern can be found in the Fourier image (see illustration in Figure 1). The more ordered a structure is, the more intense is the peak in the Fourier space at the frequency corresponding to the spacing in between the sarcomeres. We detect this special frequency location, which corresponds to a maximum in the Fourier spectrum, and we decide to label the subarea as ordered only if

the value of this peak is above a certain threshold that will be defined by the user, but that is then rather insensitive to the window size, and the global mean intensity in the image. The location of the maximum relatively to a reference axis gives us access to the orientation of the pattern $\theta$, angle of the sarcomeres with the x-axis. All sub-sections are evaluated, and 2 main parameters are given for the whole image: the percentage of ordered sub-sections and the standard deviation on $\theta$ (only calculated on the ordered sub-areas). They are respectively given by the order and dispersion parameters in Table 1. The size of sub-sections and the threshold can be adjusted by the user if necessary. To suits all needs, the initial organization is automatically found, but a dedicated user interface is proposed for fine tuning (Supplementary Figure 2). Finally, a polar histogram recapitulating organization dispersion is presented (Figure 2).

\subsection{Proof of concept:}

To assess the proper function of the proposed analysis, different cellular conditions and labelled proteins were evaluated. The myosin-light chain $2 \mathrm{v}(\mathrm{mlc} 2 \mathrm{v})$ is a protein constituting sarcomeres, thus involved in the contractile function. This protein was immuno-labelled on hiPSC-CM from two different hiPSC lines (Figure 2). As expected, the MorphoScript successfully detects a striated organization in both cell sources (Figure 2 A-B). We also successfully tested the software with $\square$-actinin immunolabelling, a protein also present in sarcomeres (Figure 2C). This confirms that multiple proteins can be labelled and efficiently detected. To increase the confidence and further demonstrate the large action perimeter of the software, freshly dissociated cardiomyocytes from control mice heart were also included in the analysis (Figure 2D).

The MorphoScript main purpose is to study the cell shape and intracellular contractile apparatus organization. To further evaluate the efficient detection of differences in striated organization, hiPSC-CM from the same line were maintained for 14 days in a common RPMI-B27 culture medium or in a commercial medium known to modify the hiPSC-CM cellular morphology (Pluricyte ${ }^{\mathrm{TM}}$, Ncardia) (Ribeiro, et al., 2015). We show here 
that the medium used for cell culture do influence the sarcomere organization and cellular morphology and we could measure, thanks to our software, significantly statistical differences in given parameters in between the two conditions.

For the purpose of analysis, images containing only entire cells were used to evaluate the cellular morphology (Figure 3 and Table 2). Among the tested parameters related to cell shape, only the area of spreading is found to be statistically different (in usual medium $1322(996 / 2276) \mu \mathrm{m}^{2} v$ s. 950 (831/1103) $\mu \mathrm{m}^{2}$ for cells grown in Pluricyte) (Table 2).

The contractile protein organization is then measured through the percentage of cell sub-areas with striation (the order) and their alignment (the dispersion). The organization of hiPSC-CM from both culture conditions were thus evaluated. Freshly dissociated cardiomyocytes are known to benefit from a perfect or nearly perfect striated organization and consequently constitute a useful positive control. While the order appears to be perfect in cardiomyocytes $(100(100 / 100))$, the use of the Pluricyte culture medium increased this parameter in hiPSC-CM (75 (59/78) vs. 93 $(90 / 94) \%$ in Pluricyte) (Figure 3 and Table 3). The dispersion of striation (consistent alignment) is drastically low in mouse cardiomyocytes $(0.04$ $(0.03 / 0.06))$, indicating a very high degree of homogeneity in the cellular striation (Figure 3 and Table 3). While cells in the Pluricyte medium clearly show a subpopulation with a very low dispersion, there is no statistical difference in striation dispersion $(0.46(0.36 / 0.55)$ vs. 0.28 $(0.31 / 0.45)$ ) (Figure 3 and Table 3 ). While mouse cardiomyocytes and

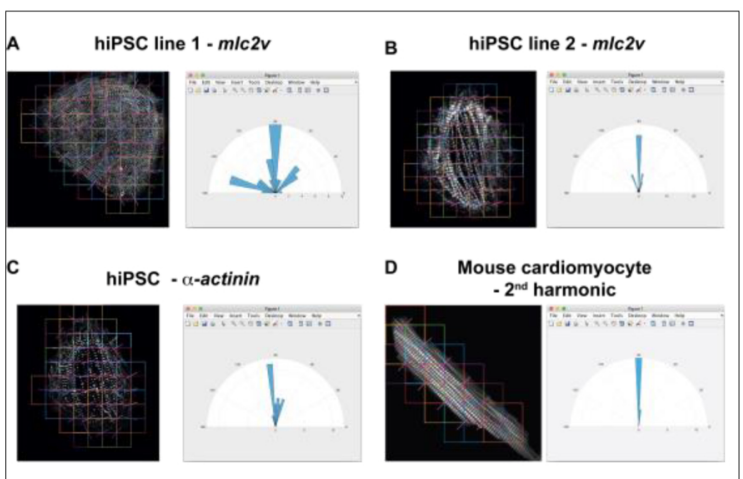

hiPSC-CM culture in the usual medium show similar striation spacing $(1.93(1.88 / 1.95)$ and $1.85(1.82 / 1.91)$ respectively), the median striation spacing is different in Pluricyte medium (1.69 (1.68/1.73)) (Table 3).
Fig. 2. The striation organization is efficiently tracked and analyzed. The MorphoScript can be successfully used regardless of the cardiomyocyte cell origin or the labelling technique used. For all examples, the cell image with sub-areas of interest are shown (left panel) along with the final polar histogram (right panel). A - B. Images of myosin light chain $2 v$ labelled hiPSC-CM from two different initial hiPSC lines. C - D. Images of actinin labelled hiPSC-CM (C) and adult mouse cardiomyocytes freshly dissociated (D)

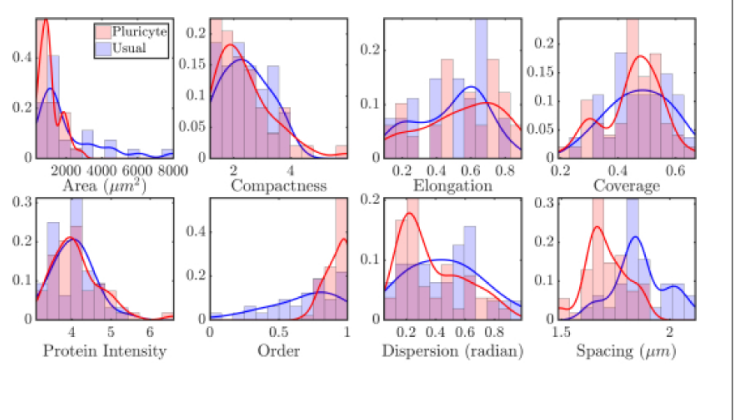

Fig. 3. Differences in the distribution of the different parameters between usual and Pluricyte culture medium. Histograms of the different probability distributions of Area $\left(\mu \mathrm{m}^{2}\right)$, Compactness, Elongation, Coverage, Normalized protein intensity, Order, Dispersion (radian), Spacing $(\mu \mathrm{m})$, with a normal kernel distribution fit (continuous lines) for usual (blue) and Pluricyte (red) culture medium. The y axis on each histogram represents the normalized density.

\section{Discussion}

The discovery of hiPSC enhanced the development of new biological research models (Takahashi, et al., 2007). Motivated by the lack of pertinent human cellular models, cardiac differentiation protocols have been developed to obtain patient specific cardiomyocytes-like cells (hiPSC-CM) (Burridge, et al., 2014; Lian, et al., 2013). Since then, many different approaches have been used to study and characterize hiPSC-CM, with notably the study of their contractions (Sala, et al., 2018). Due to their novelty, a lack of dedicated tools still exist.

The Morphoscript analysis tool aims at characterizing the shape of hiPSC$\mathrm{CM}$ and to evaluate striated structures as contractile proteins. This userfriendly analysis allows to describe the morphology of hiPSC-CM through the measure of several geometric parameters such as the cell surface, compactness, roundness, rectangularity and ellipticity parameters. If contractile proteins have been labelled, the coverage surface is also evaluated.

Previous analysis tools were designed to study animal primary cardiomyocytes which beneficiate from highly organized sarcomeric structures (Guo and Song, 2014; Pasqualin, et al., 2015). However, hiPSC$\mathrm{CM}$ usually do not recapitulate the typical rod shape of animal primary cardiomyocytes and do not show such high level of organization (Guo and $\mathrm{Pu}, 2020$; Moreau, et al., 2016). In this study, the analysis flexibility is demonstrated through several aspects since it has been tested on different hiPSC lines, different sarcomeric protein labelling and on mice primary cardiomyocytes using a specific sarcomeric detection process $\left(2^{\text {nd }}\right.$ harmonic microscopy). The mlc $2 \mathrm{v}$ and $\alpha$-actinin labelling both allowed to discriminate between the existence of a labelling and its organization (forming striated structures). To identify more local variations, smaller sub-areas can be evaluated (as long as 5 sarcomeric structures are present) 
while a more global approach can also be reached through the use of larger sub-areas. The striation orientation can be fine-tuned through a dedicated graphical user interface, allowing to correct for potentials errors made by the software. This option constitutes a huge strength allowing for a highly precise (but still fast) measurement of striation orientation.

To test whether variations in the striation can be adequately detected, a maturation medium (Pluricyte) has been used and finally compared with a primary cardiomyocyte (Ribeiro, et al., 2015). The increased percentage of organized sub-sections and the tendency to decrease the dispersion in the orientation of the sarcomeric structures suggest that the cellular maturation also occurs through changes in the contractile apparatus. hiPSC-CM cultured in Pluricyte display parameters of cellular organization between hiPSC-CM cultured in the usual culture medium and primary cardiomyocytes further demonstrating an increase but incomplete cellular maturation.

Here we describe a very powerful and convenient tool to study the cell morphology parameters and the presence and orientation of sarcomeres in hiPSC derived cardiomyocytes. The major advantage is to first identify striated pattern, regardless their consistent linear alignment. While this is great, this identification is not perfect and can requires manual correction to perfectly fit the reality. Thus, an experienced user is required to obtain the best results. Also, as previously stated, a $0.5 \mu \mathrm{m}$ minimal resolution is required to respect the Shannon criteria and appropriately measure $\sim 2 \mu \mathrm{m}$ sarcomeres. Working with much higher resolution of $0.06 \mu \mathrm{m}$, we were easily able to measure a $8.6 \%$ difference in sarcomere spacing (with Pluricyte medium for example) with high statistical power $(\mathrm{p}<0,0001)$. Finally, we do not take into account potential bias due to sample preparation method. Indeed, the fixation or the amount of calcium in the medium can affect sarcomere spacing.

\section{Conclusion}

We propose here a user friendly, flexible and highly useful analysis tool to study the morphology and striation of hiPSC-CM. The use of Matlab $\mathbb{R}$ enables a powerful analysis process that can easily evolve and adapt to user specific needs. This open source Matlab ${ }^{\circledR}$ analysis tool is freely available and can thus be combined to other analysis workflows. Finally, the use of Pluricyte $\mathbb{R}$ medium and primary cardiomyocytes demonstrated that this analysis is pertinent to identify differences in cell morphology and striation. Such analysis should thus be considered in pathologymodelling studies and even further in pharmaco-safety evaluations.

Table 1. Benchmark results of the cascade oscillators model

\begin{tabular}{|c|c|c|c|c|}
\hline & & & & Data are indicated as median (lower/upper $95 \%$ confidence interval). $*$ indicate \\
\hline Parameter & Symbol & Name & Definition & $\begin{array}{l}\text { differences between hiPSC-CM cultured in usual vs. Pluricyte medium. \# indicate } \\
\text { differences between hiPSC-CM in Pluricyte medium vs. mice cardiomyocytes, using }\end{array}$ \\
\hline Area & A & Compactness $(\mathrm{C})$ & $12 /\left(4 * \mathrm{Pi}^{*} \mathrm{~A}\right)$ & Kruskal-Wallis test. \\
\hline Perimeter & 1 & Rectangularity & $\mathrm{A} / \mathrm{Abb}$ & \\
\hline Bounding box & $A b b=l b b * w b b$ & Elongation & $1-2 * \mathrm{lbb} / \mathrm{wb}$ & ${ }_{b}$ Acknowledgements \\
\hline Minor axis ellipse & 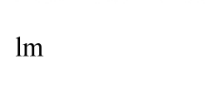 & Ellipticity & $\operatorname{lm} / \mathrm{lM}$ & $\begin{array}{l}\text { We would like to thank Dr. Xavier Nissan and the "plateforme IPS_INMG" for } \\
\text { kindly providing hiPSC lines. }\end{array}$ \\
\hline Major axis ellipse & $1 \mathrm{M}$ & & & \\
\hline
\end{tabular}

\section{Funding}

A.M was funded by the AFM Telethon and fonds Marion Elisabeth Brancher.

Table 2. Benchmark results of the cascade oscillators model

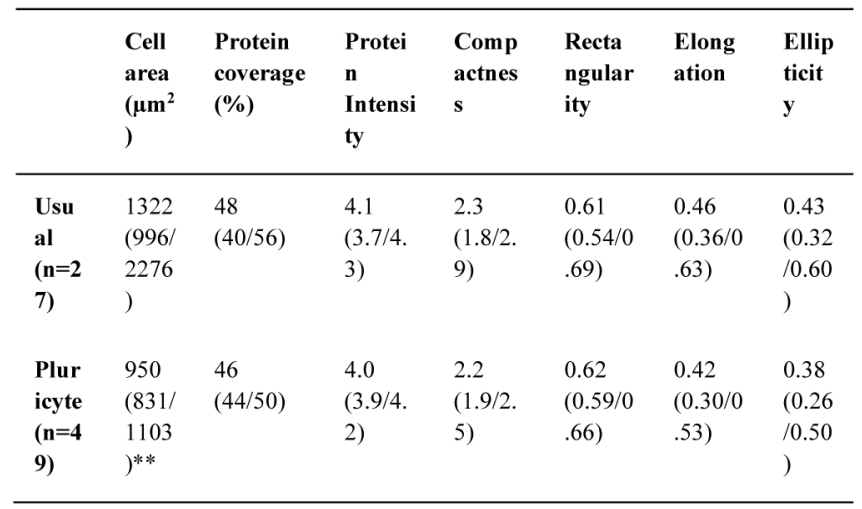

Data are indicated as median (lower/upper 95\% confidence interval). * indicate differences between hiPSC-CM cultured in usual vs. Pluricyte medium, using MannWithney test.

Table 3. Cellular striation

\begin{tabular}{|c|c|c|c|}
\hline & Order $(\%)$ & Dispersion & Spacing $(\mu \mathrm{m})$ \\
\hline $\begin{array}{l}\text { Usual } \\
(n=32)\end{array}$ & $75(59 / 78)$ & $0.46(0.36 / 0.55)$ & $1.85(1.82 / 1.91)$ \\
\hline $\begin{array}{l}\text { Pluricyt } \\
\text { e }(n=54)\end{array}$ & $93(90 / 94)^{* * * *}$ & $0.28(0.31 / 0.45)$ & $1.69(1.68 / 1.73)^{* * * *}$ \\
\hline $\begin{array}{l}\text { Cardio } \\
\text { myocyt } \\
\text { es } \\
(n=29)\end{array}$ & $\begin{array}{l}100 \\
(100 / 100)^{\# \# \#}\end{array}$ & $\begin{array}{l}0.04 \\
(0.03 / 0.06)^{\# \#}\end{array}$ & $1.93(1.88 / 1.95)^{\# \# \#}$ \\
\hline
\end{tabular}


Conflict of Interest: none declared.

\section{References}

Acimovic, I., et al. Post-Translational Modifications and Diastolic Calcium Leak Associated to the Novel RyR2-D3638A Mutation Lead to CPVT in Patient-Specific hiPSC-Derived Cardiomyocytes. J Clin Med 2018;7(11):423.

Agu, C.A., et al. Successful Generation of Human Induced Pluripotent Stem Cell Lines from Blood Samples Held at Room Temperature for up to $48 \mathrm{hr}$. Stem cell reports 2015;5(4):660-671.

Bett, G.C., et al. Electronic "expression" of the inward rectifier in cardiocytes derived from human-induced pluripotent stem cells. Heart Rhythm 2013;10(12):1903-1910. Burridge, P.W., et al. Chemically defined generation of human cardiomyocytes. Nat. Methods 2014;11(8):855-860.

Denning, C., et al. Cardiomyocytes from human pluripotent stem cells: From laboratory curiosity to industrial biomedical platform. Biochim. Biophys. Acta 2016;1863(7 Pt B):1728-1748.

Durande, M., et al. Fast determination of coarse-grained cell anisotropy and size in epithelial tissue images using Fourier transform. Physical review. E 2019;99(61):062401.

Fermini, B., et al. A New Perspective in the Field of Cardiac Safety Testing through the Comprehensive In Vitro Proarrhythmia Assay Paradigm. J. Biomol. Screen. 2015.

Gannier, F., et al. Measurements of sarcomere dynamics simultaneously with auxotonic force in isolated cardiac cells. IEEE Trans Biomed Eng 1993;40(12):12261232 .

Guo, A. and Song, L.S. AutoTT: automated detection and analysis of T-tubule architecture in cardiomyocytes. Biophys $J$ 2014;106(12):2729-2736.

Guo, Y. and Pu, W.T. Cardiomyocyte Maturation: New Phase in Development. Circ Res 2020;126(8):1086-1106.

Kadari, A., et al. Robust Generation of Cardiomyocytes from Human iPS Cells Requires Precise Modulation of BMP and WNT Signaling. Stem Cell Rev 2014

Lian, X., et al. Directed cardiomyocyte differentiation from human pluripotent stem cells by modulating Wnt/beta-catenin signaling under fully defined conditions. Nat. Protoc. 2013;8(1):162-175.

Lundy, S.D., et al. Structural and functional maturation of cardiomyocytes derived from human pluripotent stem cells. Stem Cells Dev. 2013;22(14):1991-2002.

Moisan, L. Periodic Plus Smooth Image Decomposition. Journal of Mathematical Imaging and Vision 2010;39(2):161-179.

Moreau, A., Boutjdir, M. and Chahine, M. Induced pluripotent stem-cell-derived cardiomyocytes: cardiac applications, opportunities, and challenges. Can. J. Physiol. Pharmacol. 2017;95(10):1108-1116.

Moreau, A., et al. A leaky voltage sensor domain of cardiac sodium channels causes arrhythmias associated with dilated cardiomyopathy. Scientific reports 2018;8(1):13804.

Moreau, A., et al. Biophysical, Molecular, and Pharmacological Characterization of Voltage-Dependent Sodium Channels From Induced Pluripotent Stem Cell-Derived Cardiomyocytes. Can. J. Cardiol. 2016;33(2):269-278.

Pasqualin, C., et al. Automatic quantitative analysis of t-tubule organization in cardiac myocytes using ImageJ. Am. J. Physiol. Cell Physiol. 2015;308(3):C237245 .
Ribeiro, M.C., et al. Functional maturation of human pluripotent stem cell derived cardiomyocytes in vitro--correlation between contraction force and electrophysiology. Biomaterials 2015;51:138-150.

Sala, L., et al. MUSCLEMOTION: A Versatile Open Software Tool to Quantify Cardiomyocyte and Cardiac Muscle Contraction In Vitro and In Vivo. Circ Res 2018;122(3):e5-e16.

Sleiman, Y., et al. Modeling polymorphic ventricular tachycardia at rest using patient-specific induced pluripotent stem cell-derived cardiomyocytes. EBioMedicine 2020;60:103024.

Sochacki, J., et al. Generation of urine iPS cell lines from patients with Attention Deficit Hyperactivity Disorder (ADHD) using a non-integrative method. Stem Cell Res. 2016;17(1):102-106.

Takahashi, K., et al. Induction of pluripotent stem cells from adult human fibroblasts by defined factors. Cell 2007;131(5):861-872.

Tohyama, S., et al. Distinct metabolic flow enables large-scale purification of mouse and human pluripotent stem cell-derived cardiomyocytes. Cell Stem Cell 2013;12(1):127-137. 